\title{
Comparison of frequency, field, and time domain ferromagnetic resonance methods
}

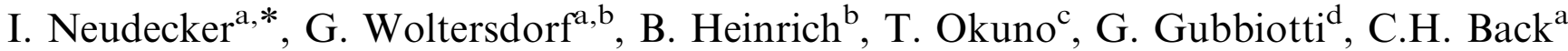 \\ ${ }^{\mathrm{a}}$ Institut fuer Experimentelle und Angewandte Physik, Universitaet Regensburg, Universitaetsstrasse 31, 93040 Regensburg, Germany \\ ${ }^{\mathrm{b}}$ Simon Fraser University, Canada \\ ${ }^{\mathrm{c}}$ Institute for Chemical Research, Koyoto University, Uji 611-0011, Japan \\ ${ }^{\mathrm{d}}$ Research Center SOFT-INFM-CNR, Università di Roma “La Sapienza," I-00185, Roma, Italy
}

Received 27 July 2005; received in revised form 26 January 2006

Available online 27 April 2006

\begin{abstract}
We present vector network analyzer ferromagnetic resonance measurements of epitaxial Fe films having a thickness of 16 monolayers. Our objective is to test the reliability of this novel frequency domain technique with respect to frequency and damping. For this purpose we compare vector network analyzer ferromagnetic resonance to pulsed inductive microwave magnetometry, time resolved magnetooptic Kerr effect (both methods in the time domain), and conventional ferromagnetic resonance (measured in the field domain) in terms of position and width of the ferromagnetic resonance. In addition, we compare the various techniques with respect to the signal to noise ratio of the raw data. All data is obtained using the same well characterized ultrathin magnetic Fe/GaAs (001) film. Finally, we demonstrate the potential of the vector network analyzer ferromagnetic resonance technique for the investigation of nano-structured magnetic elements having nonuniform magnetization configuration. The absorption spectrum of Permalloy disks with a diameter of $200 \mathrm{~nm}$ and a thickness of $15 \mathrm{~nm}$ shows up to eight distinct resonance peaks. The spatial structure of the corresponding modes was derived from numerical calculations and reveals that azimuthal modes up to the fifth order have been observed inductively.
\end{abstract}

(C) 2006 Elsevier B.V. All rights reserved.

Keywords: Ferromagnetic resonance; VNA-FMR; Ultrathin films; Permalloy disks

\section{Introduction}

For the high-frequency characterization of magnetic thin films, the method of ferromagnetic resonance (FMR) has been widely used [1]. However, there is increasing demand for the high-frequency characterization of lithographically defined micron or sub-micron sized elements used for example in novel magnetic storage schemes such as the magnetic random access memory [2-4]. Thus, novel measuring techniques are facing two problems. First, the number of spins in the devices under test are strongly reduced calling for a method with large signal to noise ratio. Second, in conventional FMR a fixed excitation frequency is used and the magnetic system is swept across the resonance

\footnotetext{
*Corresponding author. Tel.: + 49941943 4109; fax: + 499419434544.

E-mail address: ingo.neudecker@physik.uni-regensburg.de (I. Neudecker).
}

using the external bias field. Small ferromagnetic elements, however, are often not free of domains and it is of particular interest to study the dynamic response of such systems while conserving the domain structure. Here, the novel technique of vector network analyzer ferromagnetic resonance (VNAFMR) [5-7] is very advantageous. In VNA-FMR, the microwave excitation frequency is swept at a fixed bias field, thus conserving the domain state.

This paper is organized as follows. First we give a very brief introduction into the underlying theoretical background. In the next section we carefully analyze a test sample before we proceed to describe the different experimental setups. We then discuss the obtained data in terms of frequency dependence, line width, and signal to noise ratio (SNR). In the final section, we provide an example of VNA-FMR measurements using nano-sized magnetic disks. In particular, we show FMR spectra obtained in zero applied magnetic field. 


\subsection{Equation of motion}

The physical principle of manipulating a magnetic moment $\boldsymbol{M}$ is to exert a torque on it by applying a magnetic field pulse $\boldsymbol{H}(t)$ which results in a precessional motion of $\boldsymbol{M}$ around the local effective field $\boldsymbol{H}_{\text {eff }}$. The magnetization does not reach its equilibrium position directly but follows a helical trajectory due to energy dissipation. The description of this behavior was first introduced by Landau and Lifshitz [8] and was modified by Gilbert [9] in 1955 who introduced the phenomenological damping parameter $\alpha$ :

$\frac{\mathrm{d} \boldsymbol{M}}{\mathrm{d} t}=-|\gamma| \mu_{0}\left(\boldsymbol{M} \times \boldsymbol{H}_{\mathrm{eff}}\right)+\frac{\alpha}{\boldsymbol{M}_{\mathrm{s}}}\left(\boldsymbol{M} \times \frac{\mathrm{d} \boldsymbol{M}}{\mathrm{d} t}\right)$,

where $\gamma$ denotes the gyromagnetic ratio. The resulting precessional frequency is determined by the gyromagnetic ratio $\gamma$, the saturation magnetization $\boldsymbol{M}$, and the effective field $\boldsymbol{H}_{\text {eff }}$, which contains internal fields and the external bias field $\boldsymbol{H}_{0}$. In conventional FMR the magnetic system is exposed to microwave radiation at a fixed frequency. Sweeping an external magnetic bias field the magnetic response is driven through the resonance. Thus, FMR is measured only at some specific microwave frequencies.

In contrast, by using a VNA which excites a magnetic system via a high bandwidth waveguide, the external magnetic field (in the present setup $0-1.1 \mathrm{kOe}$ ) as well as the microwave frequency can be continuously adjusted (in this case in the range of $45 \mathrm{MHz}-20 \mathrm{GHz}$ ). The absorption of the system as a function of frequency provides information on the rf properties of the magnetic sample. Consequently, it is possible to investigate a magnetic system without changing the external magnetic bias field. Hence, dynamic properties of a magnetic system can be studied in any fixed field, allowing one to measure the dynamic response of the magnetization of samples in specific domain configurations even at zero field. The technique is only limited by the frequency range of the VNA.

In order to prove the reliability of the VNA-FMR technique and to compare its SNR to that of other techniques we investigated the dynamic behavior of a 16 monolayer (ML) thick Fe film and compared the obtained data to pulsed inductive microwave magnetometry (PIMM), time resolved magneto-optic Kerr effect (TRMOKE) and conventional field swept FMR measurements. All measurements were performed using the same sample.

Finally, we demonstrate the advantage of the VNAFMR technique for measurements of magnetic nanostructures with nonuniform magnetization configuration by identifying the modal spectrum of permalloy disks which in remanence exhibit a flux closed vortex state. We compare the VNA-FMR data to numerical calculations.

\section{Sample properties}

Ultrathin $16 \mathrm{ML}$ thick $\mathrm{Fe}\left(\begin{array}{ll}0 & 0\end{array}\right)$ films were prepared by means of molecular beam epitaxy. Semi-insulating, epi- ready $\operatorname{GaAs}\left(\begin{array}{lll}0 & 0 & 1\end{array}\right)$ wafers were used as templates. The GaAs substrates were heated to $400{ }^{\circ} \mathrm{C}$ in ultra high vacuum for $10 \mathrm{~h}$ in order to desorb contaminants. Residual oxides and carbon were removed by reactive desorption using an atomic hydrogen source at $400^{\circ} \mathrm{C}$. After $1 \mathrm{~h}$ of hydrogen treatment the surface contamination was below the detection limit of Auger electron spectroscopy. Subsequent annealing at up to $600^{\circ} \mathrm{C}$ was monitored using reflection high energy electron diffraction until a well ordered $4 \times 6$ reconstruction appeared. The $\mathrm{Fe}$ and $\mathrm{Au}$ films were grown at room temperature from a thermal source at a base pressure of less than $2.7 \times 10^{-8} \mathrm{~Pa}$ with a deposition rate of $\sim 1 \mathrm{ML} / \mathrm{min}[10]$. The film thickness was monitored by a quartz crystal microbalance and RHEED intensity oscillations. The Fe film was capped by $20 \mathrm{ML} \mathrm{Au}$ for protection under ambient conditions.

In order to interpret the dynamic experiments it is necessary to identify the relevant terms entering the effective field in the equation of motion. The effective field $\boldsymbol{H}_{\text {eff }}$ can be derived from the free energy density of the film:

$\varepsilon_{\mathrm{tot}}=\varepsilon_{\mathrm{zee}}+\varepsilon_{\mathrm{ani}}+\varepsilon_{\mathrm{dem}}+\varepsilon_{\mathrm{exch}}$,

where $\varepsilon_{\text {zee }}$ represents the Zeeman, $\varepsilon_{\text {ani }}$ the anisotropy, $\varepsilon_{\mathrm{dem}}$ the demagnetization, and $\varepsilon_{\text {exch }}$ the exchange energy density. For uniform precession of the magnetization the exchange energy can be neglected. To derive the static equilibrium position of the magnetization only in-plane components need to be considered since both external bias field $\boldsymbol{H}_{0}$ and magnetization $\boldsymbol{M}$ are confined to the film plane. By including the anisotropy terms of the cubic crystal anisotropy of $\mathrm{Fe}$ and the uniaxial anisotropy induced by the $\mathrm{Fe} / \mathrm{GaAs}$ interface one obtains for the total energy density:

$$
\begin{aligned}
\varepsilon_{\text {tot }}= & -M_{\mathrm{s}} \mu_{0} H_{\text {bias }} \cos \left(\varphi_{M}-\varphi_{H}\right)+\frac{1}{4} K_{1} \cos ^{2}\left(2 \varphi_{M}\right) \\
& -K_{u} \sin ^{2}\left(\varphi_{M}\right)+\frac{1}{2} \mu_{0} M_{\text {eff }}^{2},
\end{aligned}
$$

with the magnetic permeability of vacuum $\mu_{0}$ and the cubic and uniaxial in-plane anisotropy constants $K_{1}$ and $K_{u}$, respectively. The effective demagnetizing field is given by

$\mu_{0} M_{\mathrm{eff}}=\mu_{0} M_{\mathrm{s}}-\frac{2 K_{\perp}}{M_{\mathrm{s}} t}$,

which differs from the saturation magnetization $M_{\mathrm{s}}$ due to the perpendicular uniaxial interface anisotropy. $K_{\perp}$ is the interface anisotropy constant and $t$ the thickness of the film [1]. The azimuthal angles of the magnetization and the external field with respect to the [1 000 -axis are denoted by $\varphi_{M}$ and $\varphi_{H}$, respectively.

The direction of the internal effective field $\boldsymbol{H}_{\text {eff }}$ and consequently the equilibrium position of the magnetization can be determined from Eq. (3) by calculating $\partial \varepsilon_{\text {tot }} / \partial M=$ 0 at zero external field $\left(\boldsymbol{H}_{0}=0\right)$ [11]. Using typical anisotropy values reported for the $16 \mathrm{ML} \mathrm{Fe} / \mathrm{GaAs}(001)$ system [12], one obtains $\varphi_{M}=-45^{\circ}$, i.e. the easy axis is parallel to the $\left[\begin{array}{lll}1 & 1 & 0\end{array}\right]$ direction. This result shows that for a $16 \mathrm{ML} \mathrm{Fe}$ film the free energy in the film plane is 

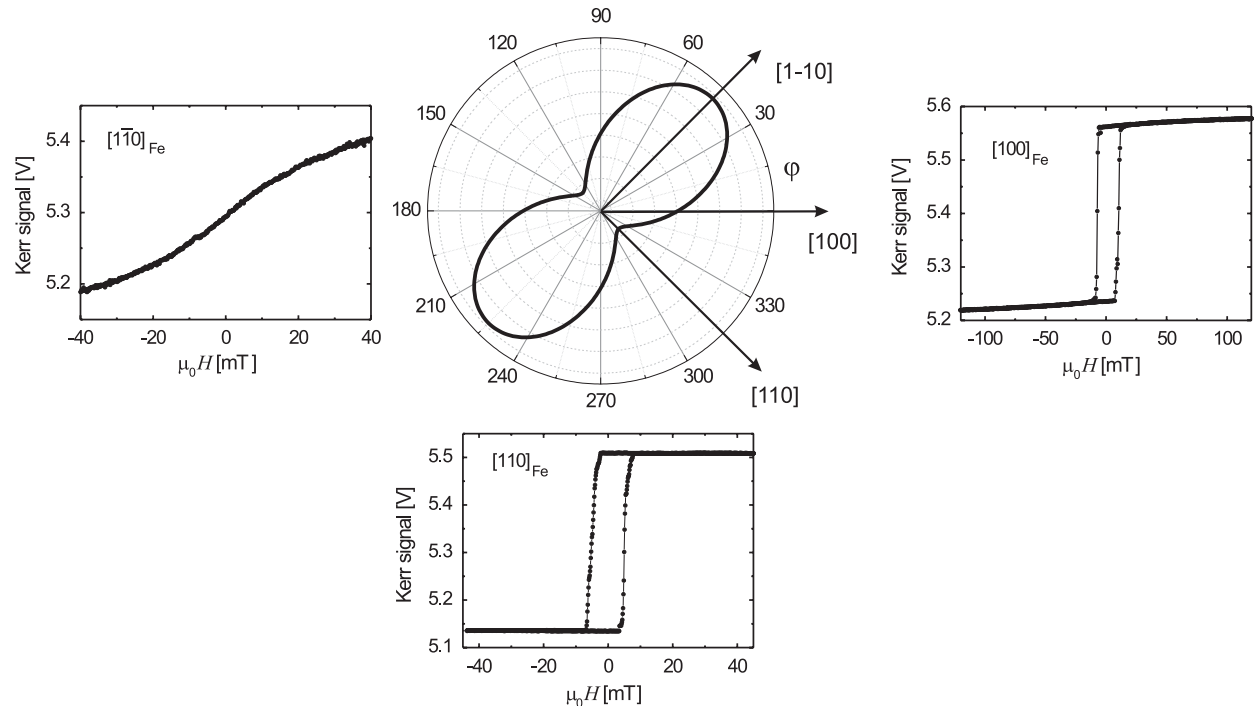

Fig. 1. The polar plot shows the free energy of the $16 \mathrm{ML}$ thick Fe film in the plane of the film as a function of the azimuthal angle $\varphi$. Corresponding MOKE loops are shown for the [1 110$]$-direction (easy axis), the [1 000$]$-direction, and the [1 $1 \overline{1} 0]$-direction (hard axis).

dominated by the uniaxial interface anisotropy, in agreement with our static Kerr effect measurements (see Fig. 1).

\section{Experimental setups}

\subsection{VNA-FMR and PIMM techniques}

The VNA-FMR and the PIMM measurements were both carried out using the same high frequency setup shown in Fig. 2 for the VNA-FMR technique. In case of the VNA-FMR measurement the vector network analyzer (Agilent PNA E8362A) serves as the source as well as the detector of the sinusoidal rf signal, which was guided via air coplanar microwave probes (Cascade Microtech Microprobes, $150 \mu \mathrm{m}$ pitch and matched to $50 \Omega$ ) to a coplanar waveguide with the magnetic system placed on top of its signal line. The vector network analyzer compares the incoming to the outgoing signals with respect to their amplitude and phase, allowing to measure the absorption of the sample as a function of frequency.

In contrast to the sinusoidal excitation used for the VNA-FMR, in the PIMM measurements a short magnetic field pulse is applied and the dynamic response of the magnetic system is monitored as a function of time [13,14]. The pulses were generated by using a pulse generator (picosecond pulse labs 10,060A) connected to one side of the setup. The rise time of the pulses was less than $65 \mathrm{ps}$ $(10-90 \%)$ with a maximum amplitude of $5 \mathrm{~V}$ and a repetition rate of $100 \mathrm{kHz}$. We used impulse excitations having a width at half maximum of $\sim 170 \mathrm{ps}$ (see inset in Fig. 4(a)). The oscillation of the magnetization was recorded after switching off the pulse. In order to read out the magnetic response the second probe was connected to a $20 \mathrm{GHz}$ sampling oscilloscope (HP 54120A).
To investigate the resonance behavior as a function of a static external magnetic field $\boldsymbol{H}_{0}$ the waveguide was mounted at the center of an electromagnet with four poles as shown in Fig. 2(a), allowing the application of magnetic fields in any direction in the film plane. The waveguide (see Fig. 2(c)) $(10 \mathrm{~nm} \mathrm{Ti}$ as adhesion layer, $200 \mathrm{~nm} \mathrm{Cu}$, and $150 \mathrm{~nm} \mathrm{Au}$ ) was fabricated on high-resistivity GaAs using optical lithography, thermal evaporation, and subsequent lift-off. The width of the center conductor was $w=90 \mu \mathrm{m}$, with a distance of $g=63 \mu \mathrm{m}$ to the ground conductors. The impedance of the waveguide was matched to $50 \Omega$. The Fe film was placed directly on top of the signal line, as can be seen in Fig. 2(c).

In order to increase the SNR of VNA-FMR and PIMM techniques reference data is taken. In doing so two different techniques are applied. First, by applying an inplane magnetic field $\boldsymbol{H}_{\text {ref }}$ perpendicular to the waveguide the magnetization is pinned parallel to the excitation field $\boldsymbol{h}_{\mathrm{rf} \|}$. As a consequence the torque in Eq. (1) is zero and precession of the magnetization is suppressed. Alternatively the reference field is applied along the waveguide. Thereby the resonance frequency is shifted to high frequencies. Subsequently this reference spectrum is subtracted from the measurement at the corresponding bias fields $\boldsymbol{H}_{0}$ to remove the background.

\subsection{The FMR technique}

The magnetic sample is mounted inside a microwave cavity which is tuned to resonance with a fixed microwave frequency of the source (klystron). The microwave absorption is measured by monitoring the power reflected from the microwave cavity using a diode detector. The sample is swept through the resonance condition by means of an external field. When the magnetic sample undergoes 

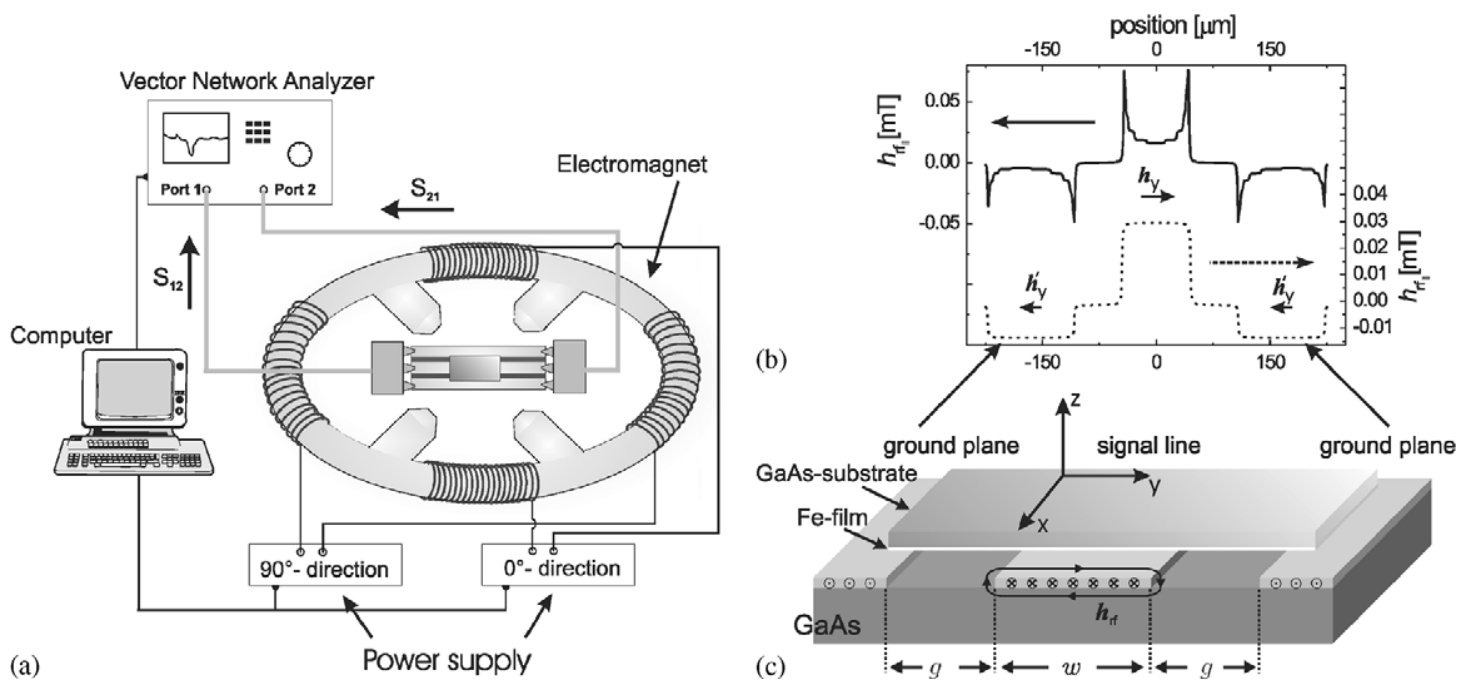

Fig. 2. (a) The experimental VNA-FMR setup with the coplanar waveguide mounted at the center of the electromagnet. It is connected to the network analyzer which acts as source and detector of the sinusoidal excitation. The Fe film on top of the coplanar waveguide is shown in detail in (c). The easy axis of the Fe film as well as the static bias field $\boldsymbol{H}_{0}$ are parallel to the x-axis. The width of the signal line and its distance to the ground conductors are $w=90 \mu \mathrm{m}$ and $g=63 \mu \mathrm{m}$, respectively. The corresponding transverse in-plane rf field profile of the waveguide is shown in (b). The dashed line is obtained from employing the Karlqvist formula $[15,16]$ by assuming uniform current distribution within the metallization. The solid line shows the realistic field distribution where the current density was calculated using an E-M software package [17].

ferromagnetic resonance the microwave losses are increased, the effective $Q$-factor of the cavity decreases, and the reflected power changes slightly.

In addition, the external magnetic field is modulated with an amplitude of $0.1 \mathrm{mT}$ at a frequency of $80 \mathrm{~Hz}$. This modulation allows lock-in detection to be used in order to increase the SNR. The measured FMR signal is proportional to the field derivative of the imaginary part of the rfsusceptibility, $\partial \chi^{\prime \prime} / \partial H$. The FMR experiments were carried out using $73,36,24$, and $10 \mathrm{GHz}$ systems.

\subsection{The TRMOKE technique}

The TRMOKE measurements are performed using a mode locked Ti-sapphire laser in a pump probe setup. The pulses of the laser with a duration of $\sim 150 \mathrm{fs}$ and a wavelength of $\sim 800 \mathrm{~nm}$ are used to generate a photocurrent across the Schottky junction at the $\mathrm{Fe} / \mathrm{GaAs}$ interface by pumping electrons inside the semiconductor [18]. This photocurrent then gives rise to a circular in-plane magnetic field pulse of approximately $2.5 \mathrm{mT}$ and a rise time of a few picoseconds. The corresponding transient field excites the magnetization. Furthermore after frequency doubling and delaying of a part of the light we use the laser pulses to probe the precession of the magnetization by means of the polar Kerr effect. Both pump and probe laser beams are focused on the sample by a polarization-conserving objective lens. The diameter of the probed area is of the order of $\sim 1 \mu \mathrm{m}^{2}$ [18].

In order to stroboscopically record the time evolution of the magnetization the delay between the pump and the probe pulses is changed. Again an external magnetic field in the direction of the easy axis of the film allows to study the dynamic properties at different bias fields.

\subsection{The excitation field amplitudes for the various techniques}

Analysis of the data in the linear regime is justified since the cone angle of the magnetic precession is small enough to apply the linearized LLG equation (see Eq. (1)). In order to validate the linear approximation, the excitation field strengths and corresponding precessional cone angles were estimated for the various techniques.

The transverse in-plane profile of the excitation magnetic field $\boldsymbol{h}_{\mathrm{rf}}$ for the waveguide excitation can be calculated by applying the Karlqvist formula $[15,16]$ and is shown in Fig. 2(b) both for uniform distribution and for realistic distribution of the current throughout the conductors calculated by using a commercial E-M software package [17] for the case of VNA-FMR. All VNA measurements were carried out with a microwave power of $1 \mathrm{~mW}$. Considering an attenuation of the setup from the source to the sample under test of $-2 \mathrm{~dB}$ a peak amplitude of $\mu_{0} \boldsymbol{h}_{\mathrm{rf}} \approx 0.2 \mathrm{mT}$ is obtained above the signal line. For the PIMM measurements the applied field pulses have a maximum amplitude of $\mu_{0} \boldsymbol{h}_{\mathrm{rf}} \approx 0.6 \mathrm{mT}$.

To estimate an upper limit of the maximum angle of the precessional motion one can compare the effective inplane anisotropy field of $\mu_{0} H_{\text {eff }}=K_{1} / M_{\mathrm{s}}+K_{u} / M_{\mathrm{s}} \approx$ $50 \mathrm{mT}$ to the amplitude of the transverse in-plane excitation field. In case of the PIMM technique one obtains a maximum cone angle in the film plane of $\theta_{\|} \approx 0.7^{\circ}$. Applying the same procedure to the maximum field of $2.5 \mathrm{mT}$ for the TRMOKE technique would result in $\theta_{\|} \approx 3^{\circ}$. In TRMOKE, however, the pulse duration is much shorter than a precessional period and the quasistatic approximation grossly overestimates the precessional cone angle. 
In the case of impulse excitation the angle of precession strongly depends on the pulse length. This effect is a consequence of the suppressed ringing when the pulse length corresponds to a half period of the magnetization precession [19].

By solving the LLG equation it can be shown that for the sinusoidal excitation one has to take into account the increase of susceptibility $\chi=160$ at resonance. The excitation field for the conventional FMR technique was approximately the same as for the VNA-FMR. Therefore the precessional cone angle for the VNA-FMR as well as for the conventional FMR is $\theta_{\|} \approx 1^{\circ}$.

The angle of precession as a function of excitation field amplitude can be derived from Eq. (1) and in the single spin approximation was found to show linear behavior in case of the $16 \mathrm{ML} \mathrm{Fe}$ film for angles exceeding the maximum cone angles in our experiments.

\section{Results and discussion}

\subsection{Resonance frequency}

First we compare the resonance frequency as a function of the external bias field $\boldsymbol{H}_{0}$ obtained from the various techniques. The experimental results are shown together with a fit to the Kittel formula in Fig. 3. Fig. 3(a) shows data from VNA-FMR, PIMM, and TRMOKE. In addition data from VNA-FMR recorded by sweeping the bias field at fixed excitation frequency is shown. Due to its larger field range, the conventional FMR data is depicted together with the VNA-FMR data in Fig. 3(b).

By solving Eq. (1) one obtains an expression for the resonance frequency:

$f_{0}=\frac{|\gamma|}{2 \pi} \mu_{0} \sqrt{H_{\text {eff }} B_{\text {eff }}}$.

$H_{\text {eff }}$ and $B_{\text {eff }}$ include the magnetic anisotropy and external fields and correspond to in-plane and out-of-plane effective fields, respectively [20]. For the particular case of the $16 \mathrm{ML} \mathrm{Fe} / \mathrm{GaAs}(001)$ magnetic film magnetized along its easy axis one obtains the Kittel formula, which describes the uniform precession of the magnetization as a function of $H_{0}$ :

$f_{0}=\frac{|\gamma|}{2 \pi} \mu_{0} \sqrt{\left(H_{\text {bias }}+M_{\text {eff }}+\frac{K_{1}}{M_{\mathrm{s}}}\right)\left(H_{\text {bias }}-\frac{2 K_{1}}{M_{\mathrm{s}}}-\frac{2 K_{u}}{M_{\mathrm{s}}}\right)}$,

where the gyromagnetic ratio is $|\gamma|=184 \mathrm{GHz} / \mathrm{T}$, the saturation magnetization is $\mu_{0} M_{\mathrm{s}}=2.1 \mathrm{~T}$, and the anisotropy constants are $K_{1}=2.7 \times 10^{4} \mathrm{~J} / \mathrm{m}^{3}$ and $K_{\perp} / t=3.3 \times$ $10^{5} \mathrm{~J} / \mathrm{m}^{3}$ (see Eq. (4)). These parameters were determined by conventional FMR [21]. The dominant uniaxial in-plane anisotropy constant was allowed to vary and used as a fitting parameter resulting in $K_{u}=-5.8 \times 10^{4} \mathrm{~J} / \mathrm{m}^{3}$.

Due to the finite size of the waveguide an inhomogeneous magnetic excitation is generated in the case of VNA-FMR and PIMM measurements. This inhomogeneity causes spin waves. The wave-vectors of the excited spin waves are given by the Fourier transform of the magnetic field distribution above the coplanar waveguide. This field distribution has a maximum at $k_{\max }=\pi / \Delta y \sim 3.5 \times 10^{4} \mathrm{~m}^{-1}$ ( $\Delta y$ is the corresponding step size for the Fourier transform). The field distribution was calculated from Maxwell's equations (see Fig. 2(b)). The amplitude of the field Fourier transform decays to $50 \%$ of its maximum value at $k_{50 \%} \sim 10^{5} \mathrm{~m}^{-1}$. As discussed by Counil et al. [22] the generation of spin waves affects both, the measured resonance frequency and the line width. According to reference [22] the resulting shift of the resonance frequency is given by

$\delta f\left(k_{\max }, \varphi_{k}\right)=\frac{1}{2}\left[f_{\mathrm{s}}\left(k_{\max }, \varphi_{k}\right)-f_{0}\right]$,

where $\varphi_{\mathrm{k}}$ is the angle of the wave-vector with respect to the magnetization. An expression for the spin wave frequency $f_{\mathrm{s}}\left(k_{\max }, \varphi_{\mathrm{k}}\right)$ was derived by Arias and Mills [23]. The frequency of the uniform precession $f_{0}$ is given by Eq. (6). In the case of the investigated $\mathrm{Fe}$ film the resulting frequency shift is maximum for $\varphi_{\mathrm{k}}=\pi / 2$ (along the $y$ direction) and corresponds to approximately $10 \mathrm{MHz}$. Therefore this effect can be neglected in our measurements.

Consequently, perfect agreement for the resonance frequencies obtained from VNA-FMR, TRMOKE, PIMM
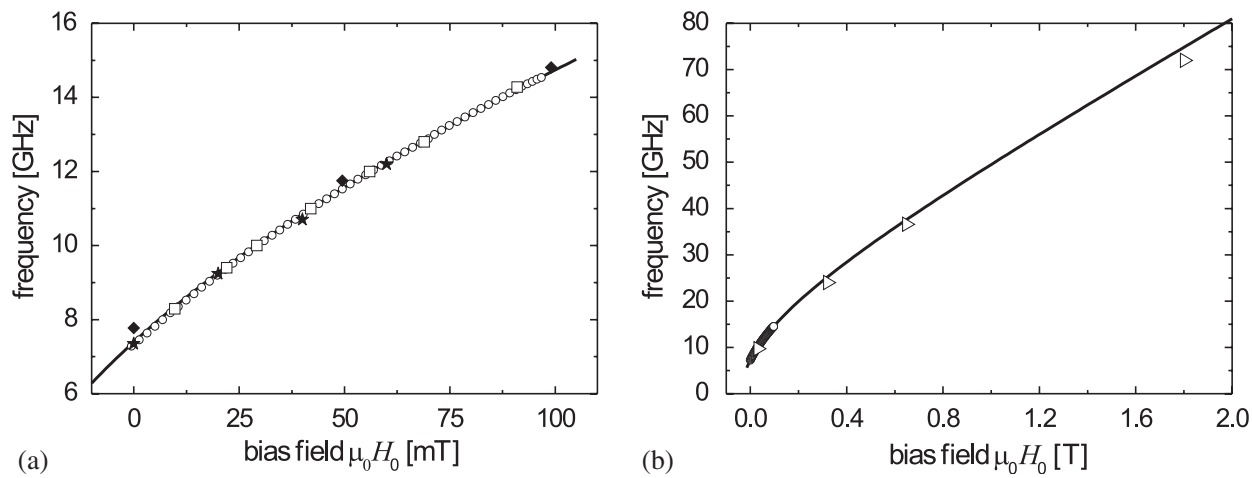

Fig. 3. (a) shows the resonance frequency as a function of bias field $H_{0}$ investigated with VNA-FMR measured by sweeping the frequency (open circles) and by sweeping the bias field (open squares). Data points from PIMM and from TRMOKE measurements are represented by stars and by diamonds, respectively. In (b) the VNA-FMR measurements (open circles) and the conventional FMR measurements (triangles) are plotted as a function of bias field. In both graphs the solid lines represent a fit to the Kittel equation (6). 
and conventional FMR techniques is expected and was indeed found (see Fig. 3).

\subsection{Magnetic relaxation}

In Fig. 5 we compare the measured effective damping constant $\alpha^{*}$ obtained by the various techniques. The two different data sets measured by the VNA techniques (field and frequency sweeps) coincide. Here, $\alpha^{*}$ is determined by fitting the data to Lorentzian line shapes and given by $\alpha^{*}=$ $|\gamma| \mu_{0} \Delta H /(2 \pi f)$ and $\alpha^{*}=4 \pi \Delta f /\left(\gamma \mu_{0}\left(B_{\text {eff }}+H_{\text {eff }}\right)\right)$ for field and frequency swept techniques, respectively. $\Delta H$ and $\Delta f$ are defined at half width and half maximum field and frequency line width, respectively (see Fig. 4(b) and (d)). From the PIMM and TRMOKE data we extract the decay time $\tau$ of the induced precessional motion which can be transformed into a damping constant $\alpha^{*}=2 /\left(\tau|\gamma| \mu_{0}\left(B_{\text {eff }}+H_{\text {eff }}\right)\right.$ ) (see Fig. 4(a) and (c)). The larger error in the PIMM data mainly arises from the timing jitter during the measurements.

Counil et al. [22] also derived an expression for the effect of line width broadening caused by the creation of spin waves:

$\Delta f\left(k_{\max }\right)=\Delta f_{0} \sqrt{1+\left(\frac{f\left(k_{\max }, \varphi_{\mathrm{k}}\right)-f_{0}}{\Delta f_{0}}\right)^{2}}$,
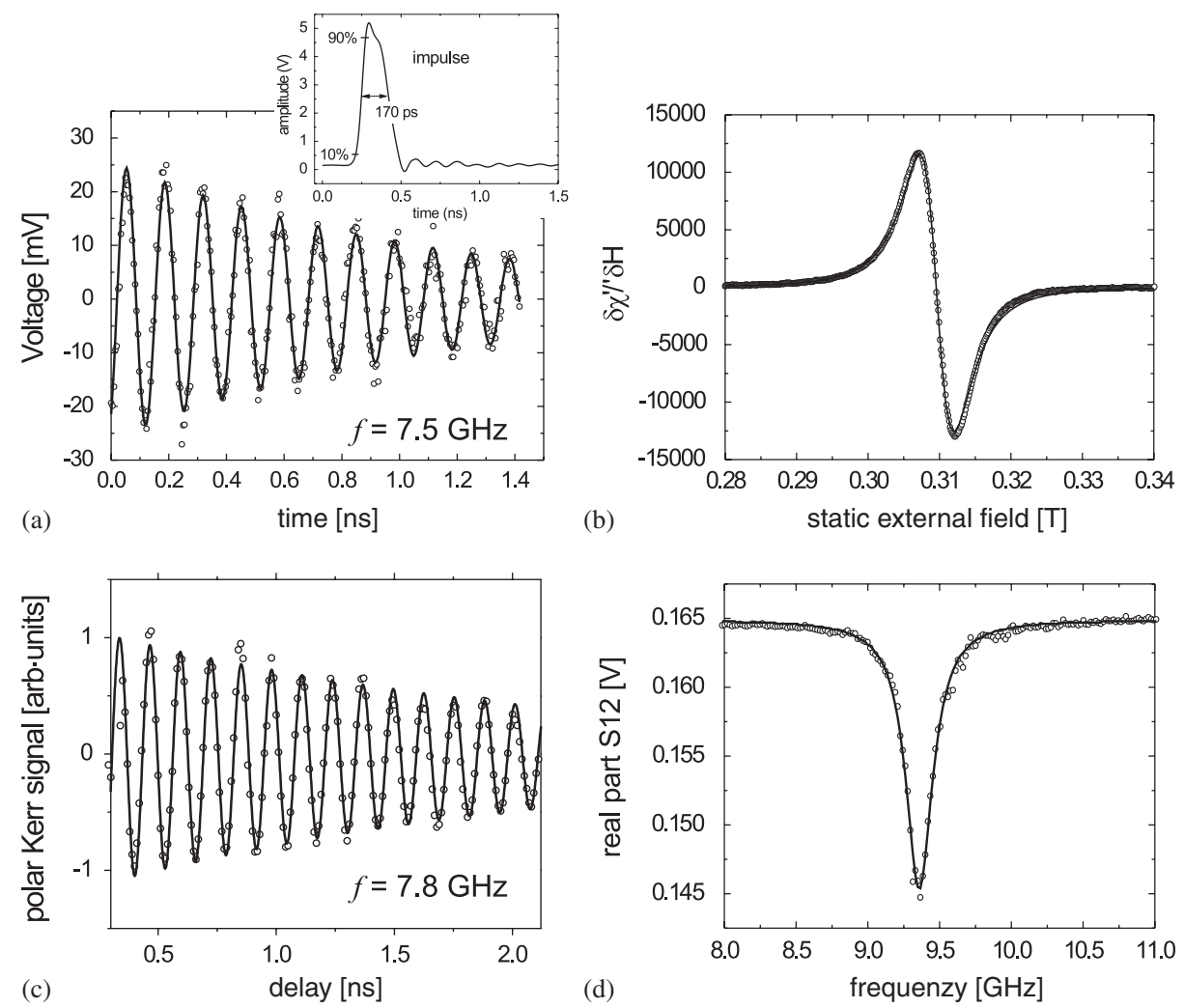

Fig. 4. The figure shows the raw data recorded using PIMM at $\mu_{0} H_{0}=0 \mathrm{~T}$ (a), conventional FMR for a microwave frequency of $9.5 \mathrm{GHz}$ (b), TRMOKE technique at $\mu_{0} H_{0}=0 \mathrm{~T}$ (c), and VNA-FMR at $\mu_{0} H_{0}=21 \mathrm{mT}$ (d). In all pictures the open circles denote the measured data points and the lines correspond to curves obtained from fits to the raw data. The inset in figure (a) shows the applied field pulse for the PIMM measurements. The rise time of 65 ps was measured from $10 \%$ to $90 \%$ of the pulse amplitude. where $\Delta f_{0}$ is the line width arising in an uniform excitation field. The corresponding line width broadening for the $16 \mathrm{ML} \mathrm{Fe}$ film is approximately $1 \mathrm{MHz}$ for $\varphi_{\mathrm{k}}=\pi / 2$ and can be neglected.

The measured values of the effective damping constant $\alpha^{*}$ are almost two times bigger than the intrinsic value $\alpha$ expected for bulk Fe [24]. The contribution from magnetic inhomogeneities to the effective damping parameter can be deduced from a decay of $\alpha^{*}$ as a function of bias field [25]. This effect is only observable in a large range of magnetic bias fields, as can be seen in Fig. 5(b). Evidently the contribution of inhomogeneities to the measured damping constant is rather weak and cannot entirely explain the observed deviation from the intrinsic bulk value. It is more likely that this enhancement is caused by the modified electronic structure of the ultrathin film compared to the bulk in combination with impurity and interface scattering. Safonov and Bertram have shown that electron-magnon scattering due to impurities and defects can result in enhanced Gilbert damping [26].

The damping observed in the TRMOKE data lies $20 \%$ below the values observed by all other techniques. The most likely reason for this effect is that the probed area in TRMOKE is much smaller than in any of the other techniques $\left(1 \mu \mathrm{m}^{2}\right)$. On this length scale the magnetic properties of the sample are more homogeneous than on a 

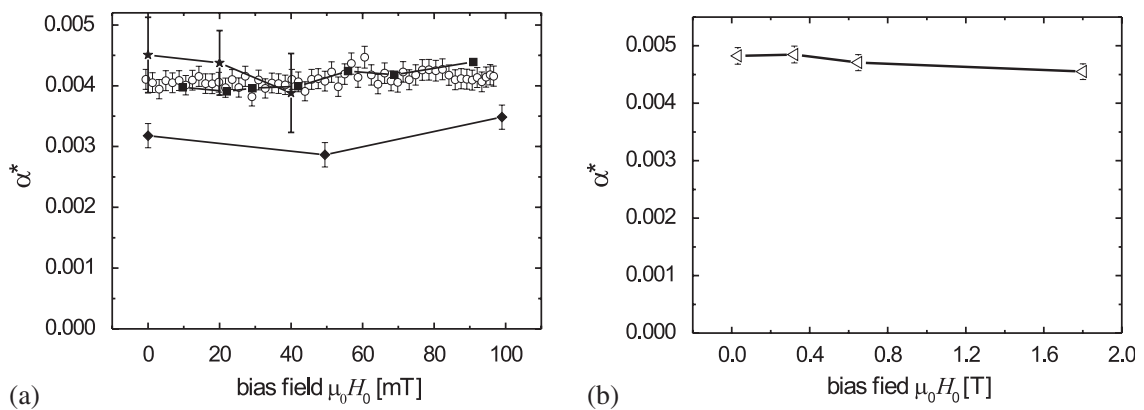

Fig. 5. (a) The effective damping constant $\alpha^{*}$ as a function of the bias field investigated using VNA-FMR by sweeping the frequency (open circles) and by sweeping the bias field (squares). Data from PIMM and from TRMOKE are represented by stars and by diamonds, respectively. (b) shows data from conventional FMR measurements as a function of the bias field. The error bars ( $4 \%$ for VNA-FMR, $7 \%$ for TRMOKE, $12 \%$ for PIMM, and $3 \%$ for FMR) account for uncertainties arising from fitting the raw data (see Fig. 4).

Table 1

The probed areas (for $16 \mathrm{ML}$ film thickness), the number of spins in units of Bohr magnetons $\mu_{\mathrm{B}}$ within the probed volume, the absolute signal to noise ratios, and the detection limit for the different techniques

\begin{tabular}{lllll}
\hline Technique & Probed area $\left[\mathrm{mm}^{2}\right]$ & Number of spins $\left[\mu_{\mathrm{B}}\right]$ & SNR (absolute) & Detection limit $\left[\mu_{\mathrm{B}}\right]$ \\
\hline PIMM (at $\left.\mu_{0} H_{0}=0 \mathrm{mT}\right)$ & 0.36 & $1.5 \times 10^{14}$ & $<10$ & $1.5 \times 10^{13}$ \\
FMR (at $24 \mathrm{GHz})$ & 2.0 & $8.5 \times 10^{14}$ & $\sim 90$ & $9.4 \times 10^{12}$ \\
TRMOKE (at $\left.\mu_{0} H_{0}=0 \mathrm{mT}\right)$ & $2.0 \times 10^{-7}$ & $8.5 \times 10^{7}$ & $<11$ & $7.7 \times 10^{6}$ \\
VNA-FMR $\left(\right.$ at $\left.\mu_{0} H_{0}=60 \mathrm{mT}\right)$ & 0.36 & $1.5 \times 10^{14}$ & $\sim 40$ & $3.8 \times 10^{12}$ \\
\hline
\end{tabular}

scale of $1 \mathrm{~mm}^{2}$ and thus the effect of line width broadening due to inhomogeneities is weaker.

\subsection{Signal to noise ratio}

In Table 1 the SNR extracted from the measurements shown in Fig. 4 and the corresponding probed areas are summarized for all techniques. The SNR normalized to the number of Bohr magnetons $\mu_{\mathrm{B}}$ for the PIMM measurements lies clearly below the values of the other techniques. The TRMOKE technique had by far the highest SNR per $\mu_{\mathrm{B}}$ due to the small area which was probed. The VNAFMR has the highest SNR per $\mu_{\mathrm{B}}$ of the inductive nonlocal techniques.

To achieve the mentioned SNR with the VNA-FMR technique the intermediate frequency (IF) bandwidth was set to $70 \mathrm{~Hz}$ and the signal was averaged over five measurements. The time needed to record one curve (including reference and probe measurement) was roughly $100 \mathrm{~s}$.

\subsection{VNA-FMR on nano-structured Permalloy disks}

To show the potential of the VNA-FMR for the investigation of nano-structured magnetic systems the dispersion spectrum of a $(2 \times 2) \mathrm{mm}^{2}$ sized array of cylindrical Permalloy $\left(\mathrm{Ni}_{81} \mathrm{Fe}_{19}\right)$ disks was measured. By using the VNA-FMR the magnetic response can be investigated at a constant external magnetic field. It is therefore possible to measure the response of the disks both in the vortex and in the saturated state. The disks have a diameter of $D=200 \mathrm{~nm}$, a thickness of $t=15 \mathrm{~nm}$, and a separation of $s=200 \mathrm{~nm}$. The same experimental setup as for the $16 \mathrm{ML}$ thick Fe film shown in Fig. 2(a) was used. Again the external bias field was applied in the plane of the disks parallel to the waveguide. The dispersion spectrum of the disks was derived by numerical calculation based on the subdivision of a particle into small cells and the development of a dynamical matrix approach [27] which allows simultaneous determination of the frequency and the dynamical magnetization profile of all the normal modes of the disk. In this model dipolar, Zeeman, and exchange interactions are considered. For further details concerning fabrication of the sample and the theoretical method, see Ref. [27].

By means of hysteresis loops measured with static Kerr effect the vortex annihilation field was found to be $\mu_{0} H_{\text {ann }}=70 \mathrm{mT}$ (see Fig. 6). The calculated frequencies are shown together with data from VNA-FMR measurements in Fig. 7. In the vortex state up to 8 distinct 
modes were observed by means of VNA-FMR (see also Fig. 8(a)), which show very good agreement with the calculated frequencies. The type and spatial structure of the modes was derived from the calculations and is labelled by ( $n=$ number of azimuthal nodes, $m=$ number of radial nodes) for the vortex state and BA (backward like) and DE (Damon Eshbach like) for the saturated state.

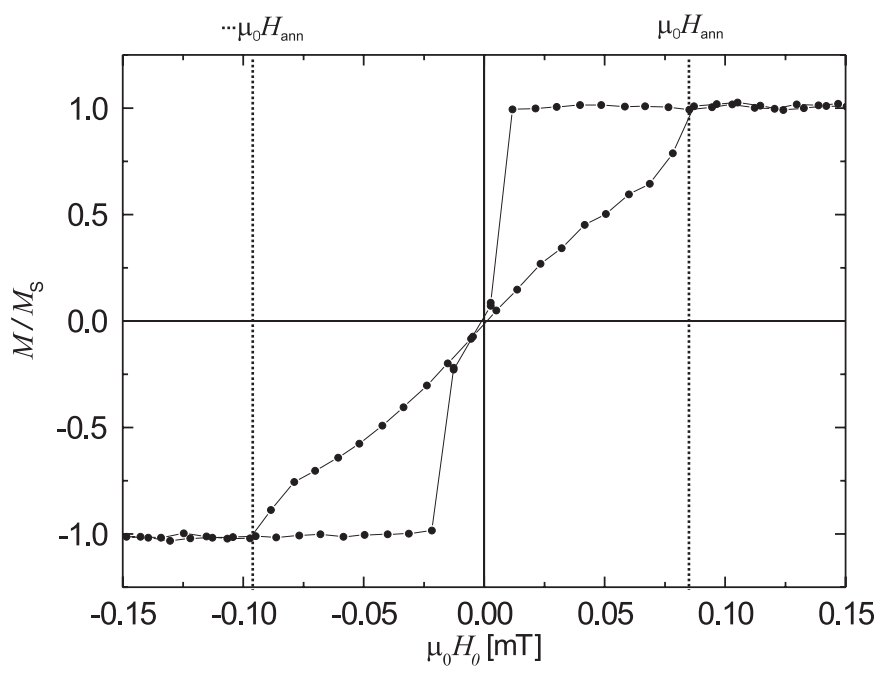

Fig. 6. The hysteresis loop for the Py disks with a diameter of $200 \mathrm{~nm}$ and a thickness of $15 \mathrm{~nm}$ recorded by static Kerr measurements. The vertical dotted line denote the vortex annihilation field at $\mu_{0} H_{\text {ann }}= \pm 70 \mathrm{mT}$.

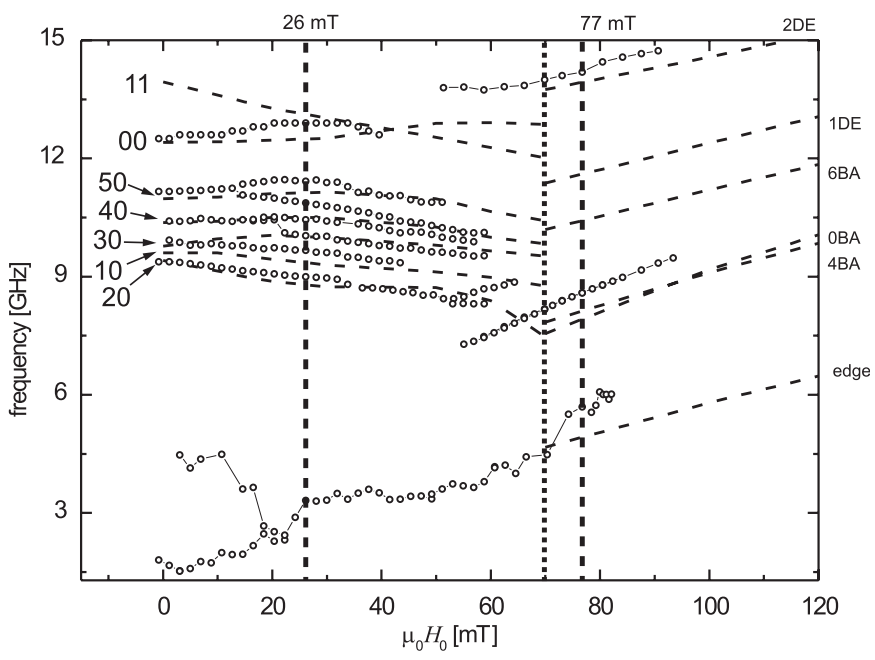

Fig. 7. Experimental data from VNA-FMR (open circles) together with calculated frequencies (dashed lines) for the Permalloy disks as a function of the external bias field $H_{0}$. The vertical doted line at $\mu_{0} H_{0}=70 \mathrm{mT}$ assigns the transition from the vortex to the saturated state. The calculated lines are labeled by ( $n=$ number azimuthal nodes, $m=$ number of radial modes) for the vortex state and by BA (backward like) and DE (Damon Eshbach like) for the saturated state with the corresponding number of nodal lines. The vertical dashed lines at $\mu_{0} H_{0}=26 \mathrm{mT}$ and $77 \mathrm{mT}$ indicate the positions where corresponding raw data is shown in Fig. 8.
When looking at the VNA-FMR data, above the vortex annihilation field one can find in the raw data a dominant mode at $f=8.6 \mathrm{GHz}\left(\mu_{0} H_{0}=77 \mathrm{mT}\right)$ (see Fig. $8(\mathrm{~b})$ ), which was identified from calculations to be the fundamental BA mode. Absorption peaks arising from ferromagnetic resonance were identified due to their dependence on the external magnetic bias field. In addition, the second DE mode and an edge localized mode was observed by means of VNA-FMR. All modes show very good agreement with the calculations and with the experimental Brillouin light scattering (BLS) results previously obtained for the same sample. A detailed description of the modes and the corresponding BLS measurements are beyond the scope of this paper and can be found in [27].

Although good agreement between the VNA-FMR data and the calculated frequencies was found, the discrepancies between the experimentally determined dispersions of the magnetic modes and the calculated frequencies likely come from coupling of the rf-field to the different modes, which is relevant for VNA-FMR. Since, however, the extent of the disk array of $(2 \times 2) \mathrm{mm}^{2}$ is larger than the width of the signal line of the waveguide $(90 \mu \mathrm{m})$ the rf-field is not in the plane of the dots throughout the whole array. The rf-field has a strong out-of-plane component near the edges of the waveguide. Therefore the measurements are not only sensitive to modes with an even number of azimuthal nodes, as expected for pure in-plane excitation. Due to the out-of-plane component of the excitation field also modes with an odd number of azimuthal nodal lines are found (see Fig. 7).

\section{Summary and conclusions}

We compared four different techniques for the investigation of magnetization dynamics in terms of frequency, damping and signal to noise ratio. Concerning the resonance frequency we found very good agreement with theoretical expectations for all four techniques. For the measured effective damping we found good agreement between the techniques but a deviation from the intrinsic bulk value. This enhancement is probably a consequence of the modified electronic structure of the ultrathin film in combination with impurity and interface scattering.

The highest SNR per spin was obtained using TRMOKE. However, concerning the inductive techniques the highest SNR per spin was obtained using the VNAFMR technique.

In the last part of the paper the potential of VNA-FMR for the investigation of nano-structured elements with nonuniform magnetization configuration was demonstrated. Due to the large SNR per spin and the fact that the external magnetic field is not required to be changed during one measurement, the dispersion spectrum of the disks could be recorded both in the vortex and in the saturated state. 

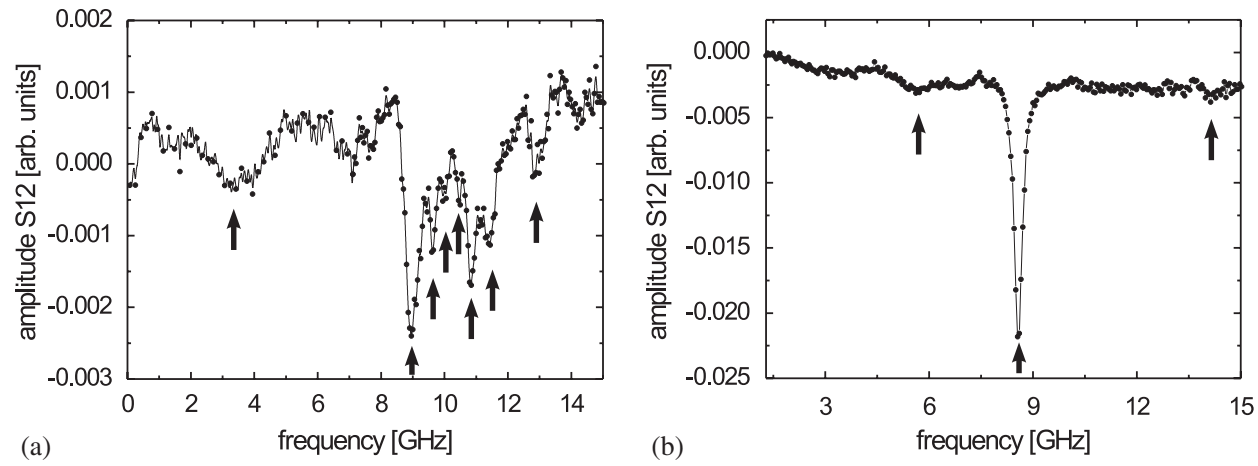

Fig. 8. Raw data for the VNA-FMR measurements of the Py disks. In the vortex state the spectrum shows 8 distinct absorption peaks at $\mu_{0} H_{0}=26 \mathrm{mT}$ (a). Above the vortex annihilation field three distinct peaks are observed at $\mu_{0} H_{0}=77 \mathrm{mT}$ (b).

\section{Acknowledgements}

Financial support by the DFG through the Priority Programme 1133 "Ultrathin Fast Magnetization Processes" is gratefully acknowledged.

\section{References}

[1] B. Heinrich, J. Bland, Ultrathin Magnetic Structures II, Springer, Berlin, 1994.

[2] S.S.P. Parkin, K.P. Roche, M.G. Samant, P.M. Rice, R.B. Beyers, J. Appl. Phys. 85 (1999) 5828.

[3] Documentation of IBM and Infineon about the research on MRAM technology. For details see webpage: 〈http://www.almaden.ibm.com/ st $/ \mathrm{magnetism} / \mathrm{ms} / \mathrm{mtj}-\mathrm{ram} / \mathrm{h}$.

[4] J. Akerman, Science 308 (2005) 508.

[5] M.B. An, D. Olligs, C. Fermon, Phys. Rev. Lett. 91 (2003) 137204.

[6] M. Bailleul, D. Olligs, C. Fermon, Appl. Phys. Lett. 83 (2003) 972.

[7] G. Counil, J.-V. Kim, K. Shigeto, Y. Otani, T. Devolder, P. Crozat, H. Hurdequint, C. Chappert, J. Magn. Magn. Mater. 272-276 (2004) 290.

[8] L. Landau, E. Lifshitz, Physik. Z. Sowjetunion 8 (1995) 153.

[9] T.L. Gilbert, Phys. Rev. 100 (1995) 1243.

[10] T.L. Monchesky, B. Heinrich, R. Urban, K. Myrtle, M. Klaua, J. Kirschner, Phys. Rev. B 60 (1999) 10242.

[11] S.V. Vonsovskii, Ferromagnetic Resonance, Pergamon Press, Oxford, 1966.
[12] M. Brockmann, M. Zölfl, S. Miethaner, G. Bayreuther, J. Magn. Magn. Mater. 198-199 (1999) 384.

[13] W. Dietrich, W.E. Proebster, P. Wolf, IBM J. Res. Develop. 4 (1960) 189.

[14] T.J. Silva, C.S. Lee, T.M. Crawford, C.T. Rogers, J. Appl. Phys. 85 (1999) 7849.

[15] O. Karlqvist, Trans. R. Soc. Technol. Stockholm 86 (1945) 1.

[16] J.C. Mallinson, The Foundations of Magnetic Recording, second ed., Academic Press, San Diego, 1993.

[17] High Frequency Electromagnetic Software, available at 〈http:// www.sonnetsoftware.com $\rangle$.

[18] Y. Acremann, M. Buess, C.H. Back, M. Dumm, G. Bayreuther, D. Pescia, Nature 414 (2001) 51.

[19] M. Bauer, R. Luposnik, J. Fassbender, B. Hillebrands, Appl. Phys. Lett. 76 (2000) 2758.

[20] B. Heinrich, J.F. Cochran, AinP 42 (1993) 523.

[21] R. Urban, G. Woltersdorf, B. Heinrich, Phys. Rev. Lett. 87 (2001) 217204.

[22] G. Counil, J.-V. Kim, T. Devolder, C. Chappert, J. Appl. Phys. 95 (2004) 5646

[23] R. Arias, D.L. Mills, Phys. Rev. B 60 (1999) 7395.

[24] Z. Frait, D. Fraitová, J. Magn. Magn. Mater. 15-18 (1980) 1081.

[25] Z. Celinski, B. Heinrich, J. Appl. Phys. 70 (1991) 5935.

[26] V. Savonov, H.N. Bertram, J. Appl. Phys. 94 (2003) 529.

[27] L. Giovannini, F. Montoncello, F. Nizzoli, G. Gubbiotti, G. Carlotti, T. Okuno, T. Shinjo, M. Grimsditch, Phys. Rev. B 70 (2004) 172404. 